\title{
Heavy quarkonium production at STAR
}

\author{
Daniel Kikoła* for the STAR Collaboration \\ Warsaw University of Technology/Lawrence Berkeley National Laboratory \\ E-mail: kikoladif.pw.edu.pl
}

\begin{abstract}
Potential models and lattice QCD calculations predict that measurement of quarkonium production provides insights into thermodynamic properties of Quark-Gluon Plasma, QGP. QGP is expected to be created in relativistic heavy ion collisions at RHIC energies. If the QGP is formed, the quarkonium production in nucleus-nucleus collisions is expected to be suppressed relative to $p+p$ interactions. The suppression in these models is caused by screening of the binding potential between quark and antiquark in QGP; a process similar to the Debye screening in a classic electromagnetic plasma. The magnitude of the suppression depends on the quarkonium binding energy and the energy density of the medium, which can be related to the temperature of the medium. Consequently the suppression pattern of the quarkonium production in nucleus-nucleus collisions would give an estimate of a temperature of the produced medium. On the other hand, there are other effects which may modify the quarkonium production rates, both attributed to "ordinary" nuclear matter (so-called cold nuclear matter effects e.g. parton shadowing, an absorption by nuclear matter or hadronic co-movers) and QGP formation (e.g. $J / \psi$ production via statistical coalescence of charm and anti-charm quarks). The suppression due to cold nuclear matter effects can be measured in $\mathrm{d}+\mathrm{Au}$ collisions and consequently a systematic measurement of quarkonium production in $\mathrm{p}+\mathrm{p}, \mathrm{d}+\mathrm{Au}$ and nucleus-nucleus interactions is required to quantify the suppression due to QGP formation.
\end{abstract}

In this article we report measurements of $J / \psi$ production and suppression in Au+Au collisions as well as $\Upsilon$ in $\mathrm{p}+\mathrm{p}$ and $\mathrm{d}+\mathrm{Au}$ collisions at $\sqrt{s_{\mathrm{NN}}}=200 \mathrm{GeV}$ at STAR. The suppression is quantified by nuclear modification factor $R_{A A}$ which is the ratio of the production in $\mathrm{Au}+\mathrm{Au}$ (or $\mathrm{d}+\mathrm{Au}$ ) collisions to the production in $p+p$ interactions scaled by corresponding number of binary collisions. Presented results are compared with predictions of theoretical models for quarkonium production and interaction with the nuclear matter.

35th International Conference of High Energy Physics - ICHEP2010,

July 22-28, 2010

Paris France

${ }^{*}$ Speaker. 


\section{Introduction}

The suppression of $J / \psi$ production due to screening of the binding potential of charm and anticharm quarks in hot and dense matter is a classic signature of the Quark Gluon Plasma formation [1] and it has been intensively studied in previous years. The NA50 and NA60 experiments at CERN-SPS (at $\sqrt{s_{\mathrm{NN}}}=17.3 \mathrm{GeV}$ ) observed a strong suppression of $J / \psi$ as a function of collision centrality. The results from the PHENIX collaboration at RHIC show that the $J / \psi$ suppression at mid-rapidity is similar to that observed at SPS energies [2], although the energy density and temperature reached at RHIC are much higher than at SPS. In addition, the suppression at RHIC at forward rapidity is stronger than at mid-rapidity. Such a pattern suggests that additional processes, such as recombination of charm quarks, sequential dissociation of exited charmonia states or feeddown from $b$-hadron, compensate for the effect of the color screening. In addition, the suppression due to so-called cold matter effects (e.g. parton shadowing, an absorption by nuclear matter and hadronic co-movers) is neither well understood nor established with satisfactory precision (for example, there are a few shadowing parametrization which differ significantly [3]).

The measurement of $\Upsilon$ suppression is expected to be less sensitive to cold matter effects. Firstly, the cross-section for absorption by interaction with hadronic co-movers is negligible [5]. Secondly, the amount of shadowing (or anti-shadowing) is smaller than in the case of $J / \psi$ since $\Upsilon$ production proceeds with higher momentum transfer $Q^{2}$ and the evolution of parton distribution functions with $Q^{2}$ reduces the effect of shadowing/anti-shadowing [6]. A difference between shadowing parameterizations decreases with increasing $Q^{2}$ as well, therefore the systematic error on cold matter effects evaluation is smaller. In addition, the primordial bottom quark production is small at RHIC energies and $\Upsilon$ production via recombination is expected to be negligible. Consequently bottomonia provide a rather direct probe of the quarkonium dissociation due to color screening in QGP.

\section{STAR detector and data analysis}

We studied $J / \psi$ and $\Upsilon(1 \mathrm{~S}+2 \mathrm{~S}+3 \mathrm{~S})$ production at mid-rapidity using STAR Time Projection Chamber (TPC) and Barrel Electromagnetic Calorimeter (BEMC). We reconstructed both $J / \psi$ and $\Upsilon$ through the di-electron decay channels. The TPC is the main STAR subsystem and it is designed to reconstruct the tracks of charged particles, giving precise information about momentum and ionization energy loss $(d E / d x)$. The TPC covers a pseudorapidity range of $-1<\eta<1$. The BEMC is a sample calorimeter with full azimuthal coverage, surrounding the TPC. It is divided in 120 modules of 40 towers each ( 20 in $\eta, 2$ in $\phi$ ). The energy resolution in the towers is $\frac{d E}{E} \sim \frac{16 \%}{\sqrt{E}}$. Electrons are identified with the BEMC using a ratio of energy deposited in the calorimeter to the track momentum measured in the TPC - in the case of electron the ratio is $\approx 1$. The BEMC was also used in $\Upsilon$ trigger for $p+p$ and $\mathrm{d}+$ Au collision.

$J / \psi$ in Au+Au collisions We studied $J / \psi$ production in minimum-bias (0-80\% of total crosssection) $\mathrm{Au}+\mathrm{Au}$ collisions at $\sqrt{s_{\mathrm{NN}}}=200 \mathrm{GeV}$ recorded in 2007 and the dataset contains 64 million events. The electron candidates were selected based on $d E / d x$ from the TPC together with $p / E<2$ cut where $p$ is a track momentum and $E$ is the energy deposited in a single BEMC tower matched to a given track. To ensure precise reconstruction of the track momentum and ionization energy loss, 
quality cuts were applied: we required at least 25 hit points for a track in the TPC and the track's distance of closest approach to the primary event vertex to be less than $1 \mathrm{~cm}$. In order to improve signal-to-background ratio we selected tracks with transverse momentum $p_{T}>1.2 \mathrm{GeV} / \mathrm{c}$.

$\Upsilon$ in $p+p$ and $\mathbf{d}+$ Au collisions $\quad$ The $\Upsilon$ trigger consists of two parts and its detailed description can be found in [8]. The first stage is a hardware trigger which reacquires a single BEMC tower energy above a given threshold $(\approx 3.5 \mathrm{GeV}$ in the case of $p+p$ and $\approx 4.3 \mathrm{GeV}$ in $\mathrm{d}+\mathrm{Au}$ collisions). The second level is a software algorithm which searches for 3 tower clusters with energy above given thresholds. An event is accepted if at least one pair of clusters is found with a large opening angle $\theta$ $(\cos (\theta)<0.5)$ and invariant mass in a range of $6-15 \mathrm{GeV} / \mathrm{c}^{2}$. Electron candidates in the off-line analysis are identified using $d E / d x$ together with $E / p$ cuts, where $E$ is a 3 tower cluster energy, and matched to clusters which triggered a given event. The results presented here are based on $p+p$ data taken in 2006 (with sampled integrated luminosity $L=7.9 \pm 0.6 \mathrm{pb}^{-1}$ ) and $\mathrm{d}+\mathrm{Au}$ collisions recoded in $2008\left(L=32 \mathrm{pb}^{-1}\right)$.

\section{Results}

Figures 1 and 2 present $J / \psi$ nuclear modification factor in Au+Au collisions measured by STAR. Figure 1 shows $R_{A A}\left(p_{T}\right)$ compared with the so-called Two-Component model [4]. The TwoComponent model is a phenomenological approach in which various aspects of the $J / \psi$ interaction with a medium were incorporated: dissociation due to color screening, hadronic phase dissociation, statistical $c \bar{c}$ coalescence, $J / \psi$ formation time effects and also $b$-hadron feed-down. Figure 2 shows the $R_{A A}$ as a function of collision centrality (represented by the number of participants, $N_{\text {part }}$ ) compared with different scenarios of the $J / \psi$ in-medium interactions. The left panel shows the comparison with the aforementioned Two-Component model while the right panel presents results from the calculations which assumed only cold nuclear matter effects (nuclear absorption and shadowing) [7] - the curves represent different shadowing parametrizations for a nuclear absorption cross section $\sigma_{a b s}=1.7 \mathrm{mb}$. Presented models are consistent with measured data. Due to limited statistics available, we are not able to distinguish between the presented scenarios of $J / \psi$ suppression.

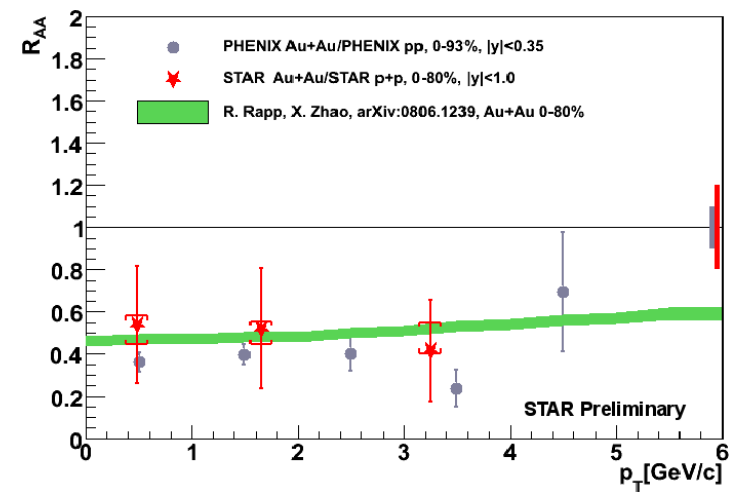

Figure 1: Nuclear modification factor as a function of transverse momentum in minimum-bias $\mathrm{Au}+\mathrm{Au}$ collisions compared to results reported by PHENIX [2] and Two-Component model [4]. The bars represent statistical error, brackets - systematic error, and the boxes about unity on the right show the $R_{A A}$ normalization uncertainty. The green band shows the uncertainties in the model.

In the case of $\Upsilon$ we found that the cross-section in $p+p$ collisions is $B r \times(d \sigma / d y)_{y=0}^{1 \mathrm{~S}+2 \mathrm{~S}+3 \mathrm{~S}=}$ $114 \pm 38$ (stat.) ${ }_{-24}^{+23}$ (syst.) pb. This is the first measurement of $\Upsilon$ production at RHIC energies. The 

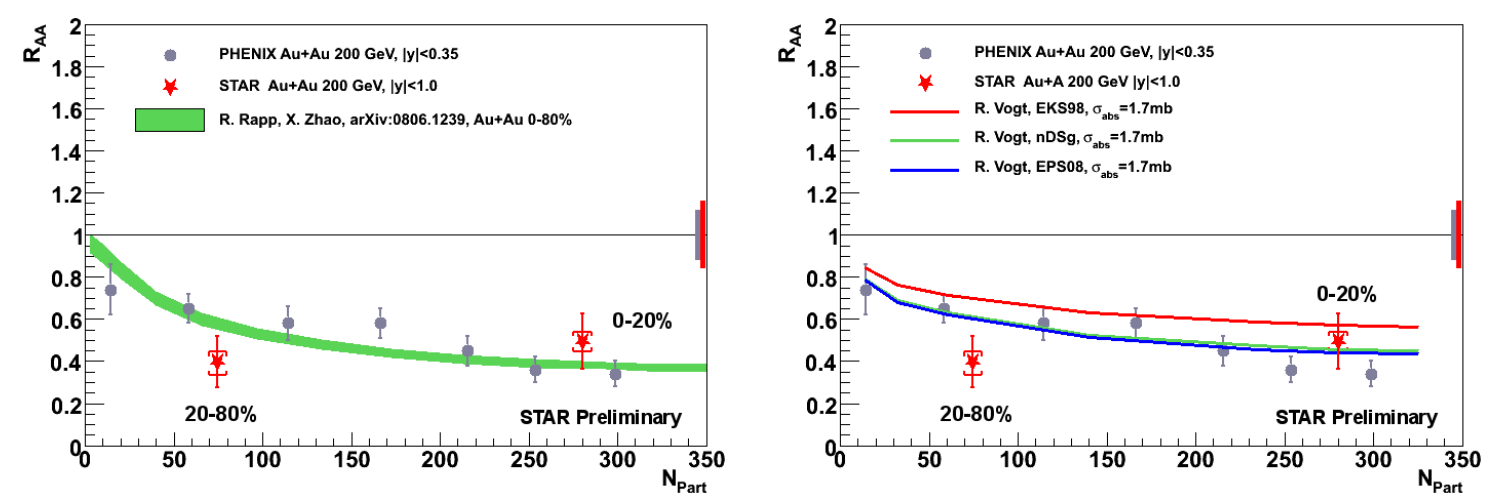

Figure 2: Nuclear modification factor as a function of centrality in Au+Au collisions compared to results reported by PHENIX [2] and the Two-Component model (left) and predictions for $R_{A A}$ from cold matter effects only (shadowing and nuclear absorption) (right panel).

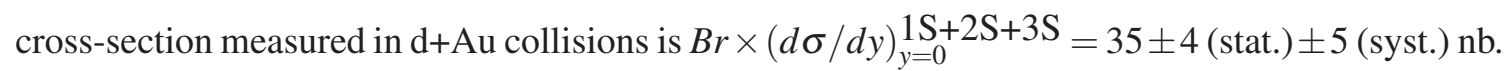
which gives the nuclear modification factor $R_{d A u}=0.78 \pm 0.28$ (stat.) \pm 0.20 (syst.). This result supports theoretical predictions that cold nuclear matter effects are not significant in the case of $\Upsilon$, although statistical and systematic errors of measured values are large.

\section{Summary}

We have reported the nuclear modification factor for $J / \psi$ in $\mathrm{Au}+\mathrm{Au}$ and $\Upsilon(1 \mathrm{~S}+2 \mathrm{~S}+3 \mathrm{~S})$ in $\mathrm{d}+\mathrm{Au}$ collisions at $\sqrt{s_{\mathrm{NN}}}=200 \mathrm{GeV}$. The strong $J / \psi$ suppression is observed and a good agreement with published results is seen although current statistical precision does not allow for discrimination between different models of $J / \psi$ in-medium interactions. The measurement of $\Upsilon$ nuclear modification factor supports theoretical expectations that cold nuclear matter effects are are not significant in the case of bottomonium. In addition, STAR recently collected a large set of $\mathrm{Au}+\mathrm{Au}$ and $p+p$ data and new precise measurements of $\Upsilon R_{d A u}$ and $J / \psi R_{A A}$ are expected soon.

Acknowledgments This work was supported in part by Polish Ministry of Science and Higher Education under grant no. 2409/B/H03/2009/37.

\section{References}

[1] T. Matsui and H. Satz, Phys. Lett. B 178 (1986) 416.

[2] A. Adare et al. [PHENIX Collaboration], Phys. Rev. Lett. 98 (2007) 232301.

[3] K. J. Eskola, H. Paukkunen and C. A. Salgado, JHEP 0904, 065 (2009).

[4] X. Zhao and R. Rapp, [arXiv:0806.1239v1]

[5] Z. w. Lin and C. M. Ko, Phys. Lett. B 503, 104 (2001).

[6] S. Liuti and R. Vogt, Phys. Rev. C 51, 2244 (1995).

[7] R. Vogt, Private communication.

[8] B. I. Abelev et al. [STAR Collaboration], Phys. Rev. D 82, 012004 (2010). 